\title{
Acoustic Phonons in Nanowires with Embedded Heterostructures
}

\author{
Hussein M. Ayedh ${ }^{1,2}$ and Andreas Wacker ${ }^{1}$ \\ ${ }^{1}$ Mathematical Physics, Lund University, Box 118, 22100 Lund, Sweden \\ ${ }^{2}$ Physics Department, Faculty of Science, Thamar University, Box 87246, Dhamar, Yemen \\ Correspondence should be addressed to Andreas Wacker, andreas.wacker@fysik.lu.se \\ Received 30 June 2011; Accepted 10 August 2011 \\ Academic Editor: Raymond Whitby
}

Copyright ( $) 2011$ H. M. Ayedh and A. Wacker. This is an open access article distributed under the Creative Commons Attribution License, which permits unrestricted use, distribution, and reproduction in any medium, provided the original work is properly cited.

Acoustic phonons are studied in finite nanowires based on the elastic continuum model. The phonon dispersion relations are compared for different types of approximations both for pure nanowires and nanowire heterostructures including cubic and wurtzite materials. We find that the isotropic approximation highly underestimates the frequency of torsional modes. The impact of heterostructures on the lowest modes is rather weak and can be described by an average of the different structure parameters.

\section{Introduction}

The phonon spectrum for semiconductor nanowires is important for a variety of applications. On the one hand, nanowires are of high interest for thermoelectric applications [1, 2 ], where the energy transport by phonons deteriorates the performance [3]. Furthermore, the phonon scattering is an important feature for all sorts of electronic devices [4]. This interaction could recently be directly experimentally accessed $[5,6]$.

Currently, most calculations of the phonon spectrum are done either within the isotropic approximations for ideal (e.g., [7]) and coupled [8] quantum wires or using atomistic approaches (e.g., [3, 9]). Here, we present corresponding calculations using the commercial COMSOL package [10], which allows to treat both the correct cubic material properties and nanowires with embedded heterostructures in a straightforward way. This enables us to check the quality of the approximation by an isotropic material and the impact of heterostructures [11] on the acoustic branches, which is most important for the broadening of the zero photon line [12].

Our paper is organized as follows: in Section 2, we show how we extract the phonon dispersion relation from simulations of a finite nanowire length. Here, we consider the isotropic approximation, where one has easy access to analytical results for comparison. Section 3 focuses on the cubic material properties of GaAs wires, where we analyze the differences for the acoustic branches. Nanowire heterostructures are considered in Section 4, where we show that the acoustic branches are only weakly affected. The application to wurtzite structures, see also [13], is demonstrated in Section 5.

\section{Obtaining the Phonon Spectrum from Finite Nanowires}

Throughout this work, we apply the elastic continuum model, which treats the crystal as a continuum. Thus, the internal vibrations that give rise to the optical modes cannot be considered in this model [14]. It is valid as long as the wavelength of the phonon mode is much longer than the lattice dimension.

The linear relation between stress $T$ and strain $S$ has the general form

$$
T_{i}=\sum_{j=1}^{6} c_{i j} S_{j}, \quad(i=1,2, \ldots, 6)
$$

Here, $c_{i j}$ are the elastic stiffness constants with 36 elements in Voigt notation. These stiffness constants can be reduced depending on the symmetries of the material. Many common semiconductors having diamond structure (e.g., silicon and germanium) or zincblende structure (e.g., GaAs and InAs) 
exhibit cubic symmetry. The matrix of elastic constants for such materials has only three independent elements $[15,16]$, $c_{11}, c_{12}$, and $c_{44}$.

For isotropic approximation these three independent elastic moduli for the cubic materials can be decreased into two moduli, and they have been expressed by Lamé constants $\lambda$ and $\mu$

$$
\begin{gathered}
\lambda=c_{12}, \\
\mu=\frac{\left(c_{11}-c_{12}\right)}{2} .
\end{gathered}
$$

Then, the elastic moduli matrix in the isotropic approximation will only include both of Lamé constants as distinct constants

$$
c=\left[\begin{array}{cccccc}
2 \mu+\lambda & \lambda & \lambda & 0 & 0 & 0 \\
\lambda & 2 \mu+\lambda & \lambda & 0 & 0 & 0 \\
\lambda & \lambda & 2 \mu+\lambda & 0 & 0 & 0 \\
0 & 0 & 0 & \mu & 0 & 0 \\
0 & 0 & 0 & 0 & \mu & 0 \\
0 & 0 & 0 & 0 & 0 & \mu
\end{array}\right] .
$$

As most of the materials have their elastic properties described in terms of Young's modulus E and Poisson's ratio $v$ [17], the two Lamé constants $\lambda$ and $\mu$ can be written in terms of $E$ and $v$ [17]

$$
\begin{gathered}
\lambda=\frac{E v}{(1+v)(1-2 v)}, \\
\mu=\frac{E}{2(1+v)} .
\end{gathered}
$$

Here, we approximate the actual shape of the nanowires by a cylinder with radius $R$, length $l$, and homogeneous mass density $\rho$. The acoustic vibrational modes of an infinite cylinder can be classified into three types: longitudinal modes, torsional modes, and flexural modes [17].

The longitudinal modes represent the vibrations of the atoms around their equilibrium positions, where the displacements of the atoms are mostly parallel to the direction of the nanowire [17]. In contrast to bulk longitudinal modes, there is also a small radial component due to the Poisson's ratio. The angular frequency $\omega$ of each mode is related to its wavevector $k$ by the following relation:

$$
\omega=c k,
$$

this relation is called the dispersion relation and the proportionality constant is the mode velocity. The longitudinal modes velocity $c_{\text {long }}$ have the form

$$
c_{\text {long }}=\sqrt{\frac{E}{\rho}} .
$$

In the torsional vibrations, the radial displacement of the atoms about their equilibrium positions is perpendicular to the direction of the wave propagation. The torsional modes also have linear dispersion relation as in (5), and the torsional modes velocity $c_{\text {tor }}$ has the following form:

$$
c_{\text {tor }}=\sqrt{\frac{\mu}{\rho}} .
$$

The flexural modes are more complicated than the previous modes. The dispersion relation of the flexural modes is quadratic

$$
\omega=f_{\text {flex }} k^{2}
$$

where the proportionality factor of the flexural modes reads

$$
f_{\text {flex }}=\sqrt{\frac{E R^{2}}{4 \rho}} .
$$

Simulating a finite nanowire by COMSOL, we obtain directly the set of eigenfrequencies. From the spatial structure, see Figure 1, we determine the character of the modes and determine the wavevector $k=2 \pi / \lambda$ by a close inspection of the spatial periodicity of the mode (with period $\lambda$ ) in the wire direction. One can clearly identify the different branches from the infinite wire. Figure 2 shows the calculated lowest frequencies for $\mathrm{GaAs}$ nanowire with isotropic approximation of length $225 \mathrm{~nm}$ and radius $20 \mathrm{~nm}$. In this way, we obtain the dispersion relations (5), (8). From the data in Figure 2, we extract the velocities $c_{\text {long }}=3890 \mathrm{~m} / \mathrm{s}$ and $c_{\text {tor }}=2484 \mathrm{~m} / \mathrm{s}$. These values are very close to the theoretical values given by (6) and (7), $c_{\text {long }}=4020 \mathrm{~m} / \mathrm{s}, c_{\text {tor }}=2484 \mathrm{~m} / \mathrm{s}$. The extracted proportionality factor of the flexural modes $f_{\text {flex }}=$ $4.7 * 10^{-5} \mathrm{~m}^{2} / \mathrm{s}$ also agrees roughly with the theoretical value given by (9), $f_{\text {flex }}=4 * 10^{-5} \mathrm{~m}^{2} / \mathrm{s}$. We conclude that our procedure allows to obtain reliable data for the dispersion relations.

\section{Isotropic Approximation versus Cubic Nanowire}

In this section, the isotropic symmetry, which manifests the same result at any direction, will be compared to the actual cubic symmetry of GaAs. As is well known and mentioned above, the cubic symmetry has three distinct elastic moduli whereas the isotropic material has only two independent elastic constants. The question that arises currently is how the dispersion relations of phonon modes, in material with cubic symmetry, will differ from those that have been displayed previously with isotropic symmetry. The COMSOL simulation tool allows for a treatment of both types of symmetries, which are directly compared in this section. For this purpose, we study the phonon modes in GaAs nano-wires along [001] direction. In Figure 3, one can easily notice the difference between the dispersion relations of the two kinds of symmetries. The longitudinal modes have similar dispersion relations in both, and they have almost identical longitudinal velocities in the both symmetries. The dispersion relation of the flexural modes in the two types of symmetries are similar at lower frequencies, but they 
TABLE 1: The density $(\rho)$ and the elastic properties Young's modulus (E) and Poisson's ratio ( $\nu$ ) of materials of zincblende structure that considered in this work as isotropic material.

\begin{tabular}{lccc}
\hline Material & $\rho\left(\mathrm{kg} / \mathrm{m}^{3}\right)$ & $\mathrm{E}(\mathrm{GPa})$ & $\nu$ \\
\hline $\mathrm{GaAs}^{\mathrm{a}}$ & $5318^{\mathrm{b}}$ & 85.9 & 0.31 \\
$\mathrm{AlAs}^{\mathrm{a}}$ & 3598 & 83.5 & 0.32 \\
$\mathrm{InAs}^{\mathrm{c}}$ & $5667^{\mathrm{b}}$ & 51.4 & 0.35 \\
$\mathrm{InP}^{\mathrm{d}}$ & 4810 & 61.1 & 0.36 \\
\hline
\end{tabular}

: Elastic properties are from [18].

b: [17].

c: Elastic properties are from [19].

d: [20].

TABLE 2: The elastic moduli of materials with cubic symmetry.

\begin{tabular}{lccc}
\hline Material & $c_{11}(\mathrm{GPa})$ & $c_{12}(\mathrm{GPa})$ & $c_{44}(\mathrm{GPa})$ \\
\hline $\mathrm{GaAs}^{\mathrm{a}}$ & 118.8 & 53.8 & 59.4 \\
$\mathrm{AlAs}^{\mathrm{a}}$ & 120.2 & 57 & 58.9 \\
\hline
\end{tabular}

${ }^{\mathrm{a}}:[21]$.

manifest a divergence at high frequencies. More noticeable, the torsional modes exhibit a significant difference in their dispersion relations, as shown in Figure 3. For the cubic material, we extract $c_{\text {tor }}=3343 \mathrm{~m} / \mathrm{s}$, while the isotropic approximation provides $c_{\mathrm{tor}}=\sqrt{\mu / \rho}=2484 \mathrm{~m} / \mathrm{s}$ as addressed above. This difference can be elucidated by the fact that the Lamé coefficients extracted from the material parameters in Table 1 by (4) satisfy $\lambda=c_{12}$ and $\mu=\left(c_{11}-c_{12}\right) / 2$ for the cubic parameters from Table 2. Now, the isotropic approximation, see (3), requires $c_{44}=\left(c_{11}-c_{12}\right) / 2$, which, however, does not hold exactly for cubic materials; see Table 2 . In contrast, the observed velocity $c_{\text {tor }}$ for the torsional modes equals $\sqrt{c_{44} / \rho}$ in this case. As $c_{44}>\left(c_{11}-c_{12}\right) / 2$, the isotropic approximation underestimates the torsional velocity in [001] direction.

\section{Acoustic Phonons in Nanowire Heterostructure}

Now, we consider acoustic phonons in nanowire heterostructures in order to compare the results to those of simpler pure nanowires. We consider structures, where the wires consist of segments of different materials stacked in the wire direction as experimentally realized in [11]. Note that the interfaces between the materials are perpendicular to the wire direction in contrast to the case intensively studied by Balandin and coworkers [22], where the interfaces are parallel to the wire direction. Due to the lacking translational invariance in the wire direction, $k$ is not an exact quantum number for the phonon modes in our case. Nevertheless, we can still extract an average distance $\lambda$ between nodes or maxima in the mode profile as discussed above, and thus obtain an effective $k=$ $2 \pi / \lambda$ for comparison with the ideal wires.

We consider a nanowire of total length $225 \mathrm{~nm}$. In the middle, there is GaAs region with length $35 \mathrm{~nm}$, embedded between two barriers of AlAs of thickness $5 \mathrm{~nm}$. The outer
TABle 3: The density $(\rho)$ and the elastic moduli of materials with wurtzite symmetry.

\begin{tabular}{lcccccc}
\hline Material & $\begin{array}{c}\rho \\
\left(\mathrm{kg} / \mathrm{m}^{3}\right)\end{array}$ & $\begin{array}{c}c_{11} \\
(\mathrm{GPa})\end{array}$ & $\begin{array}{c}c_{12} \\
(\mathrm{GPa})\end{array}$ & $\begin{array}{c}c_{13} \\
(\mathrm{GPa})\end{array}$ & $\begin{array}{c}c_{33} \\
(\mathrm{GPa})\end{array}$ & $\begin{array}{c}c_{44} \\
(\mathrm{GPa})\end{array}$ \\
\hline $\mathrm{GaN}^{\mathrm{a}, \mathrm{d}}$ & $6095^{\mathrm{b}}$ & 390 & 145 & 106 & 398 & 105 \\
$\mathrm{AlN}^{\mathrm{c}, \mathrm{d}}$ & $3255^{\mathrm{b}}$ & 410 & 148 & 99 & 388 & 130 \\
\hline
\end{tabular}

a: Elastic moduli are from [23].

b: [17].

c: Elastic moduli are from [24].

$\mathrm{d}:[16]$.

regions are again GaAs. This structure constitutes the prototype of an electron resonant tunneling nanowire device. The acoustic phonons in the AlAs/GaAs/AlAs nanowire heterostructure display the same behavior as in the pure GaAs nanowire of the same length; see Figure 4(a). Very slight differences in the eigenfrequencies of the phonon modes are observed due to existence of another material, and we checked that these differences are getting larger by increasing the amount of the other material. Consequently, the dispersion relations of all phonon modes are essentially the same in a nanowire and a nanowire heterostructure. For the heterostructure, we extract the values $c_{\text {long }}=3898 \mathrm{~m} / \mathrm{s}$, $c_{\text {tor }}=2494 \mathrm{~m} / \mathrm{s}$, and $f_{\text {flex }}=4.75 * 10^{-5} \mathrm{~m}^{2} / \mathrm{s}$ in the isotropic approximation, which are slightly larger than the corresponding values for pure GaAs wires are $c_{\operatorname{long}(\mathrm{GaAs})}=3881$, $c_{\mathrm{tor}(\mathrm{GaAs})}=2484 \mathrm{~m} / \mathrm{s}$, and $f_{\mathrm{flex}(\mathrm{GaAs})}=4.7 * 10^{-5}$. The main difference can be accounted for by considering an average material of $95.556 \%$ GaAs and $4.444 \%$ AlAs, corresponding to the composition of the finite wire. Then, we expect

$$
\begin{aligned}
c_{\text {long }(\mathrm{av})} & =\frac{95.556 * c_{\text {long }(\mathrm{GaAs})}+4.444 * c_{\text {long }(\mathrm{AlAs})}}{100} \\
& =3915 \mathrm{~m} / \mathrm{s}
\end{aligned}
$$

and similarly $c_{\text {tor(av) }}=2505 \mathrm{~m} / \mathrm{s}$ and $f_{\text {flex(av) }}=4.8 *$ $10^{-5} \mathrm{~m}^{2} / \mathrm{s}$, which all slightly overestimate the increase.

Similar results have been obtained from the comparison between phonon modes in nanowire and nanowire heterostructure with the cubic symmetry. The dispersion relations of phonon modes in a cubic pure GaAs nanowire are in full accord with those in a cubic GaAs/AlAs nanowire heterostructure, as it is illustrated in Figure 4(b). The phonon modes in these two kind of nanostructures have also very small variances in their eigenfrequencies due to the existence of small part of another material which has different elastic properties.

Indium arsenide (InAs) and indium phosphide (InP) also have a zincblende structure with three independent elastic constants. But for simplicity, we have used the isotropic approximation to study the phonon modes in InAs/InP heterostructure. Then, the dispersion relations of the acoustic phonon modes in InAs/InP heterostructure have been compared to those in a pure InAs nanowire; see Figure 5. It is difficult to distinguish the difference in the frequencies of the modes in a nanowire and a nanowire heterostructure by just looking at Figure 5, due to the smallness of these variances. These small variances arise from usage of different 


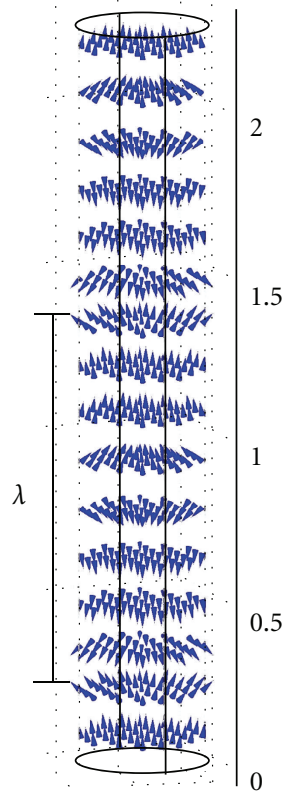

(a)

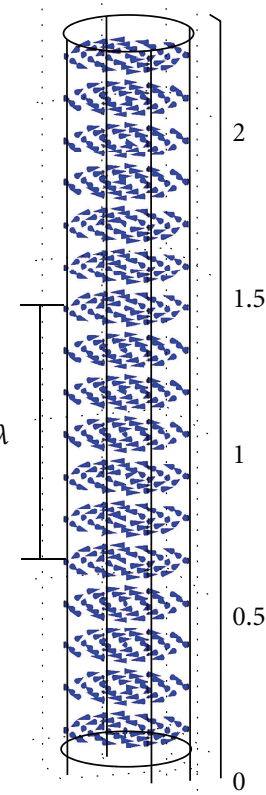

(b)

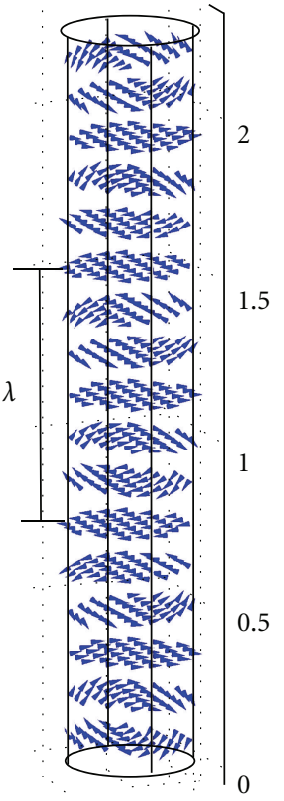

(c)

FIgURE 1: Examples of the vibrational mode shapes. (a) Longitudinal mode, (b) Torsional mode, and (c) Flexural mode. The vertical axis displays the nanowire height in units if $100 \mathrm{~nm}$.

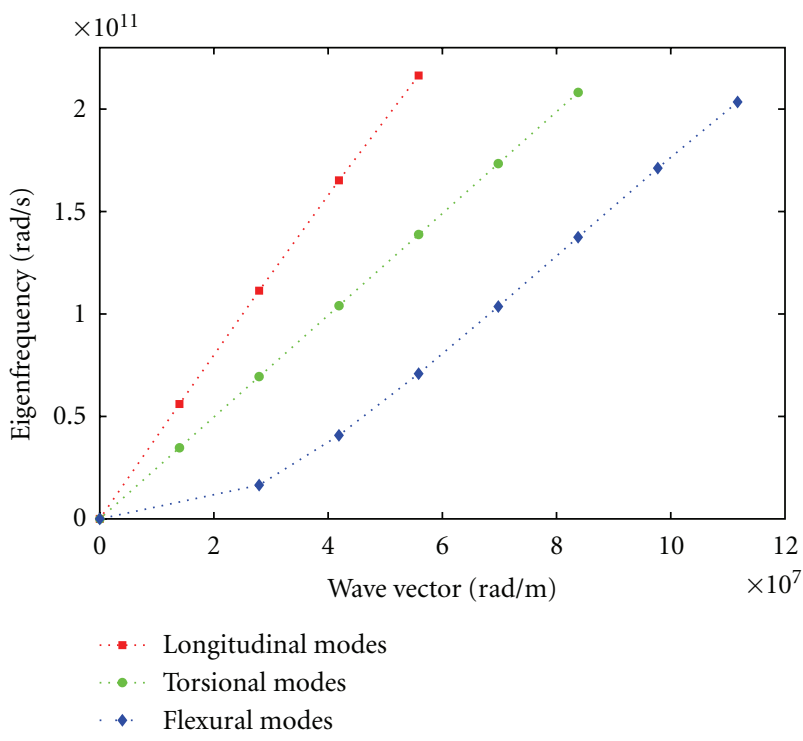

FIgURE 2: Dispersion relations of the acoustic phonons in pure GaAs nanowires with isotropic approximation.

material and it depends on the percentage contribution of each substance in the nanoheterostructure size. We extract the values $c_{\text {long }}=2960 \mathrm{~m} / \mathrm{s}, c_{\text {tor }}=1842 \mathrm{~m} / \mathrm{s}$, and $f_{\text {flex }}=$ $3.5 * 10^{-5} \mathrm{~m}^{2} / \mathrm{s}$ for the heterostructure wire. Here, the assumption of an average material provides $c_{\text {long(av) }}=2961 \mathrm{~m} / \mathrm{s}$, $c_{\text {tor(av) }}=1844 \mathrm{~m} / \mathrm{s}$, and $f_{\text {flex(av) }}=3.32 * 10^{-5} \mathrm{~m}^{2} / \mathrm{s}$. This approximation works much better for the InAs/InP material system than for the GaAs/AlAs case discussed above (except for the flexural modes, where the coefficient is difficult to extract).

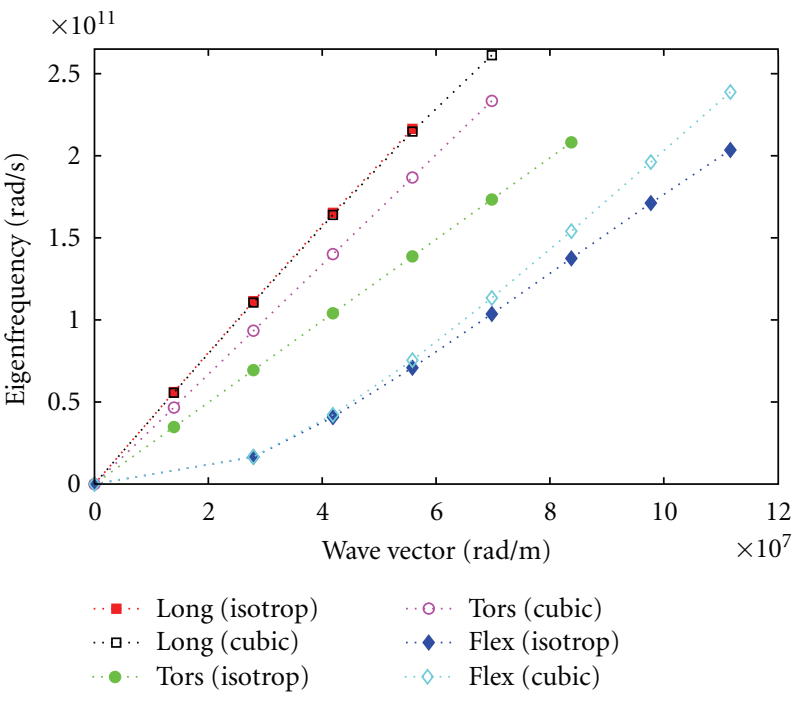

FIGURE 3: Dispersion relations of the acoustic phonons in pure GaAs nanowires with isotropic and cubic symmetries along [001] direction. The results for the longitudinal modes fall on top of each other.

Another way to prove the similarities of the acoustic phonon modes, with small wavevectors, in both pure InAs nanowire and InAs/InP nanoheterostructure are the comparisons of the strain components in both structures. Figure 6 illustrate the likeness of normal strains for the longitudinal modes and the shear strains for the flexural modes in both pure InAs nanowire and InAs/InP nanoheterostructure regardless of the discontinuities of the strain components at the interfaces between InAs and InP segments. One can see mirror reflections around $z$-axis of some modes in 


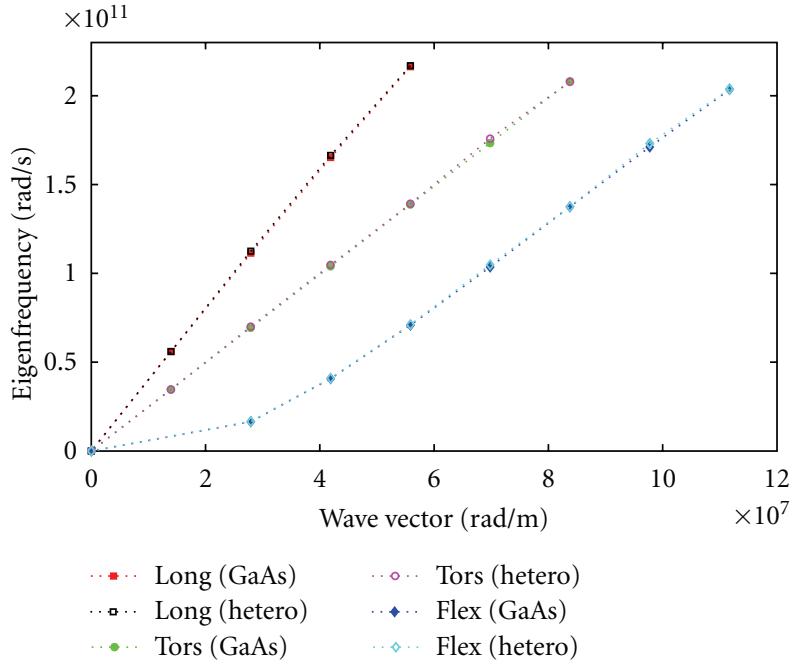

(a) Isotropic approximation

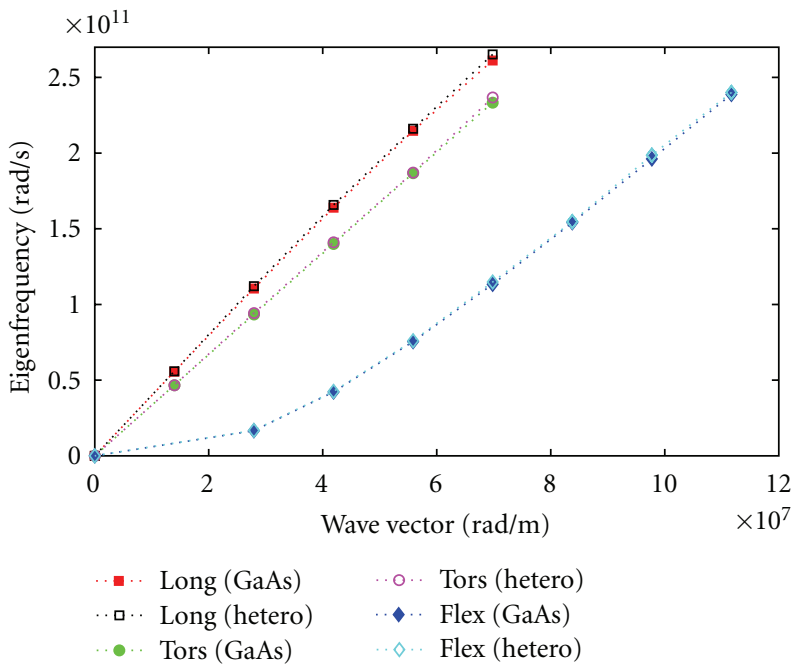

(b) Cubic symmetry

Figure 4: These figures illustrate that the phonon dispersion relations in pure GaAs nanowire and GaAs/AlAs nanowire-heterostructures are almost identical. (a) GaAs nanowire and GaAs/AlAs nanowire-heterostructure with isotropic approximation. (b) Same structures calculated with cubic symmetry. The wire length is $225 \mathrm{~nm}$ in all cases.

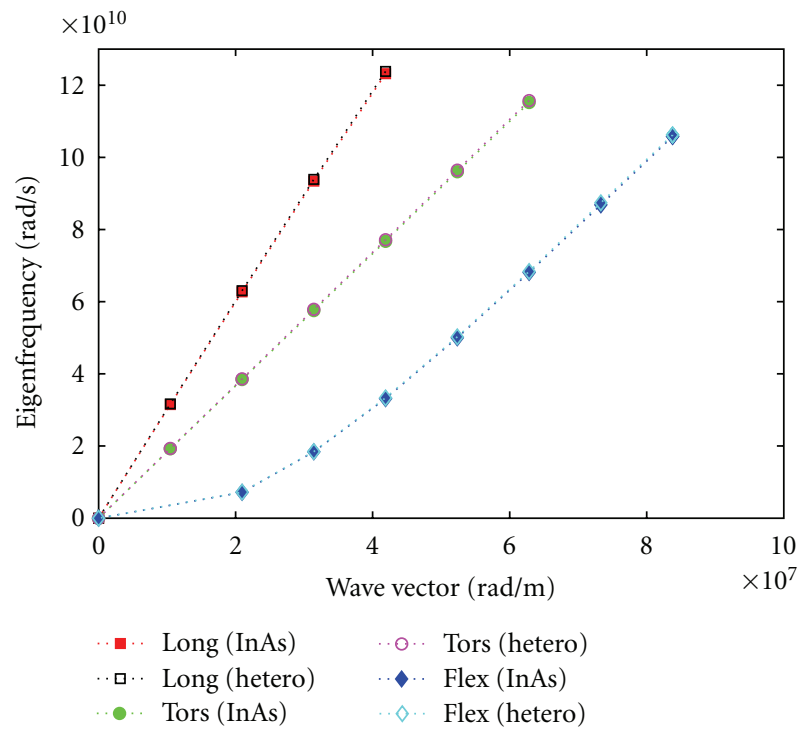

Figure 5: Dispersion relations of phonons in both InAs nanowire and InAs/InP nanowire heterostructure with the same length $300 \mathrm{~nm}$, which are almost equal to each other.

the figures, but with the same strain values. The reason is that the solver in COMSOL multiphysics is solving for the absolute value which may take positive or negative values. Taking this into account, we conclude that the strain profiles are only weakly affected by the presence of the heterostructure.

\section{Acoustic Phonons in a Nitride Heterostructure}

Another kind of structure has been used in this work, the wurtzite structure. The materials that have wurtzite struc- ture, such as Gallium nitride ( $\mathrm{GaN}$ ) and aluminum nitride $(\mathrm{AlN})$, manifest symmetry in the elastic moduli matrix with five independent constants $c_{11}, c_{12}, c_{13}, c_{33}$, and $c_{44}$ [14, 16, 17]; see Table 3 . The motivation of studying acoustic phonons in plenty of materials with different structures is to explore whether acoustic phonons might show different behavior in different structures. The dispersion relations of acoustic phonon modes have been drawn in a pure GaN nanowire in Figure 7(a) and a GaN/AlN nanoheterostructure in Figure 7(b). In these structures the longitudinal and torsional modes have linear dispersion relations, as it is observed above for the isotropic and zincblende materials. The flexural modes have two components due to bending of the nanostructure toward ( $x$-axis) and ( $y$-axis). While these are exactly degenerate for cubic materials addressed in preceding sections, these two flexural components become nondegenerate for higher frequencies in the pure GaN nanowire as well as in the GaN/AlN heterostructure. The acoustic phonon eigenfrequencies in the GaN/AlN heterostructure are slightly higher than those in a pure GaN nanowire, and the approximation of an average material again slightly overestimates the trend.

\section{Conclusion}

The phonon dispersion relation, $\omega(k)$, that holds for an infinite wire, has been extracted from COMSOL calculations in finite nanostructures made of different III-V semiconductors such as GaAs, InAs, and GaN. We find that the dispersion relation of torsional modes has changed significantly during the switching between the isotropic and cubic symmetries, while the longitudinal modes are not influenced at all for a [001] wire direction. For heterostructures, we demonstrated, that the phonon spectrum is only weakly modified and the strain components do not vary essentially. This suggests that 


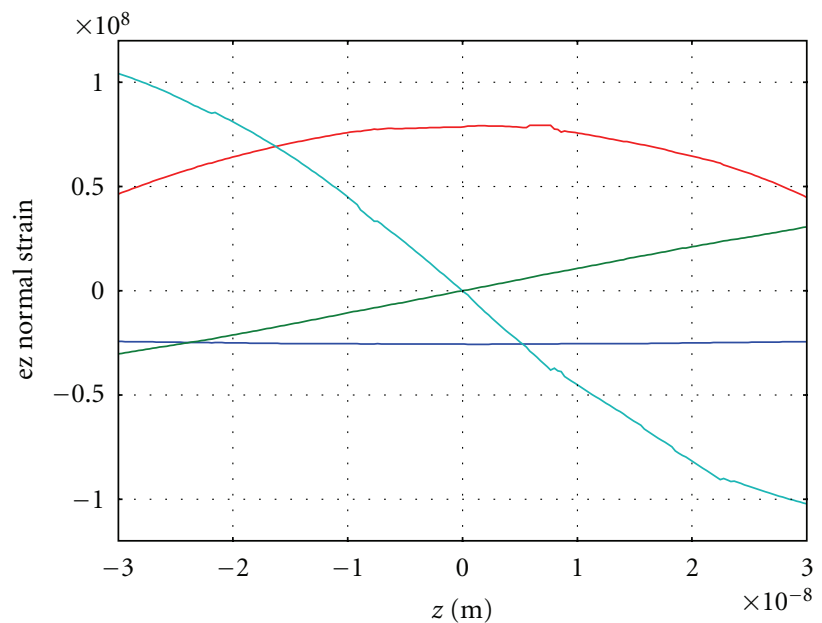

(a) Pure nanowire (longitudinal)

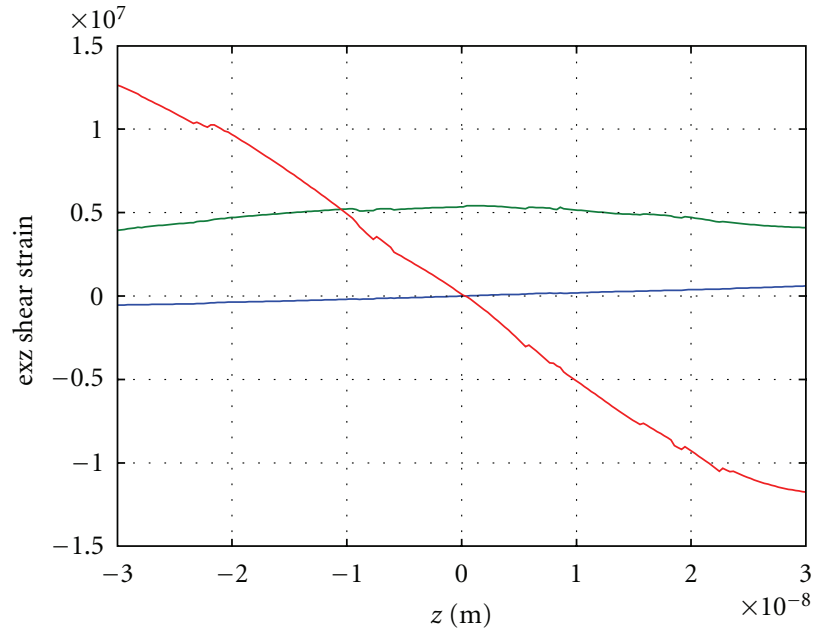

(c) Pure nanowire (flexural)

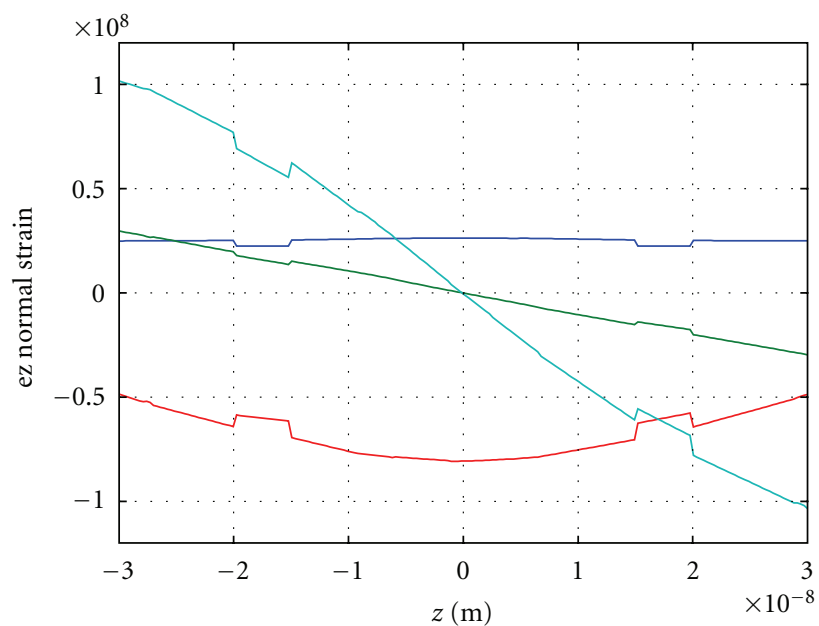

(b) Heterostructure (longitudinal)

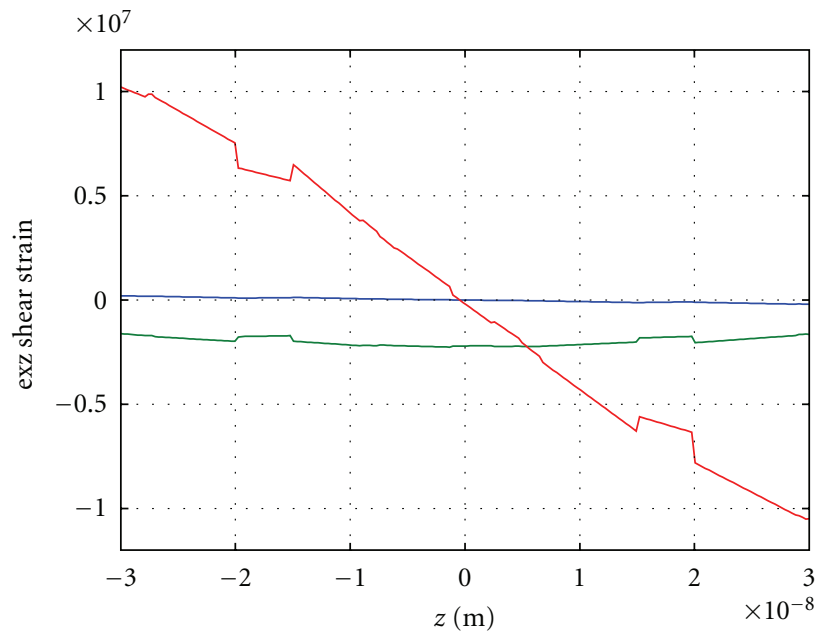

(d) Heterostructure (flexural)

FIGURE 6: The normal and shear strains $e_{z}$ and $e_{x z}$ of the lowest longitudinal and flexural modes respectively in a cross-section taken in the middle of an InAs nanowire and an InAs/InP nanowire heterostructure with $l=60 \mathrm{~nm}$ along $z$-axis, where the region shown includes two barriers and the quantum well in the heterostructures (calculations in isotropic approximation).

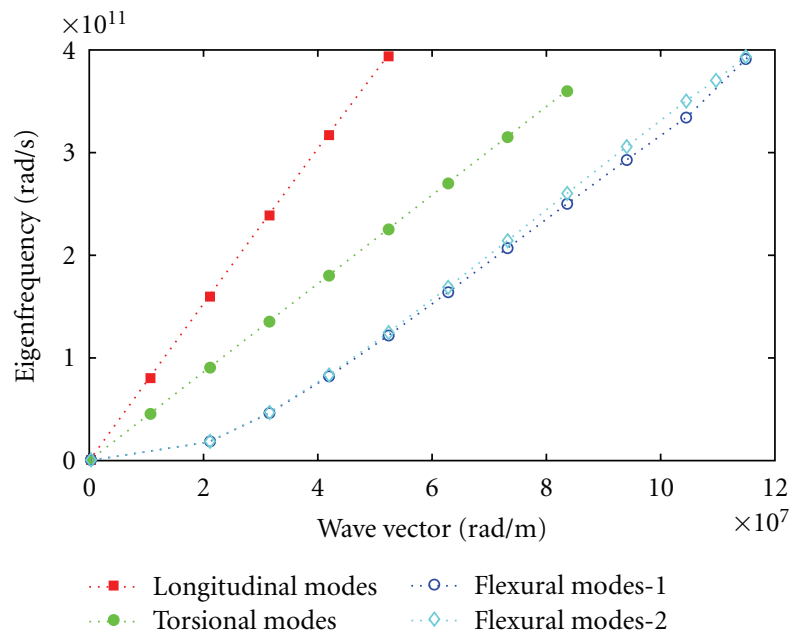

(a) GaN nanowire

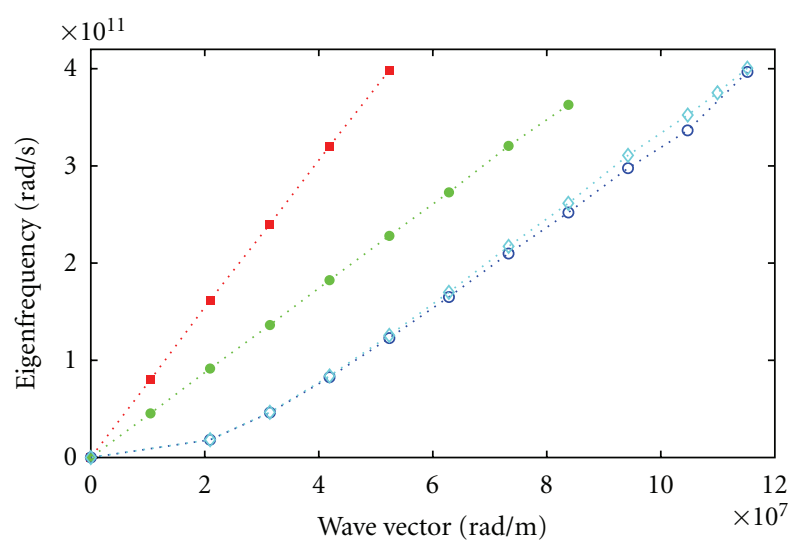

-... Longitudinal modes $\quad$.. o. Flexural modes-1

-.. Torsional modes $\quad \cdots \diamond$. Flexural modes-2

(b) GaN/AlN

FIgURE 7: Dispersion relation of phonons in a GaN nanowire and a GaN/AlN nanowire heterostructure with the same length $300 \mathrm{~nm}$ using the wurtzite symmetry. 
one can safely ignore the presence of heterostructures if the lowest phonon branches are needed in calculations for electron scattering processes.

\section{Acknowledgments}

Financial support from the Erasmus Mundus program and the Swedish Research Council (VR) is gratefully acknowledged.

\section{References}

[1] M. S. Dresselhaus, G. Chen, M. Y. Tang et al., "New directions for low-dimensional thermoelectric materials," Advanced Materials, vol. 19, no. 8, pp. 1043-1053, 2007.

[2] A. I. Boukai, Y. Bunimovich, J. Tahir-Kheli, J. K. Yu, W. A. Goddard, and J. R. Heath, "Silicon nanowires as efficient thermoelectric materials," Nature, vol. 451, no. 7175, pp. 168$171,2008$.

[3] N. Mingo, "Calculation of Si nanowire thermal conductivity using complete phonon dispersion relations," Physical Review B, vol. 68, no. 11, pp. 1133081-1133084, 2003.

[4] C. Thelander, P. Agarwal, S. Brongersma et al., "Nanowirebased one-dimensional electronics," Materials Today, vol. 9, no. 10, pp. 28-35, 2006.

[5] C. Weber, A. Fuhrer, C. Fasth, G. Lindwall, L. Samuelson, and A. Wacker, "Probing confined phonon modes by transport through a nanowire double quantum dot," Physical Review Letters, vol. 104, no. 3, Article ID 036801, 2010.

[6] P. Roulleau, S. Baer, T. Choi et al., "Coherent electron-phonon coupling in tailored quantum systems," Nature Communications, vol. 2, no. 1, 2011.

[7] M. A. Stroscio, K. W. Kim, S. Yu, and A. Ballato, "Quantized acoustic phonon modes in quantum wires and quantum dots," Journal of Applied Physics, vol. 76, no. 8, pp. 4670-4675, 1994.

[8] W.-Q. Huang, M.-L. Zou, G.-F. Huang, J.-J. Yao, and W. Hu, "Material properties dependence of ballistic phonon transmission through two coupled nanocavities," Journal of Applied Physics, vol. 105, no. 12, 2009.

[9] T. Thonhauser and G. D. Mahan, "Phonon modes in Si [111] nanowires," Physical Review B, vol. 69, no. 7, Article ID 075213, pp. 752131-752135, 2004.

[10] COMSOL, "Multiphysics user's guide," comsol 3.5 ed., 2008, http://www.comsol.com/.

[11] M. T. Björk, B. J. Ohlsson, T. Sass et al., "One-dimensional heterostructures in semiconductor nanowhiskers," Applied Physics Letters, vol. 80, no. 6, p. 1058, 2002.

[12] G. Lindwall, A. Wacker, C. Weber, and A. Knorr, "Zerophonon linewidth and phonon satellites in the optical absorption of nanowire-based quantum dots," Physical Review Letters, vol. 99, no. 8, Article ID 087401, 2007.

[13] E. P. Pokatilov, D. L. Nika, and A. A. Balandin, "Acousticphonon propagation in rectangular semiconductor nanowires with elastically dissimilar barriers," Physical Review B, vol. 72, no. 11, pp. 1-4, 2005.

[14] M. A. Stroscio and M. Dutta, Phonons in Nanostructures, Cambridge University Press, Cambridge, UK, 2001.

[15] C. Kittel, Introduction to Solid State Physics, John Wiley \& Sons, Toronto, Canada, 7th edition, 1996.

[16] P. Yu and M. Cardona, Fundamentals of Semiconductors Physics and Materials Properties, Springer, Berlin, Germany, 3rd edition, 2003.
[17] A. N. Cleland, Foundations of Nanomechanics from SolidState Theory to Device Applications, Springer, Berlin, Germany, 2003.

[18] S. Adachi, "GaAs, AlAs, and $\mathrm{Al}_{\mathrm{x}} \mathrm{Ga}_{1-\mathrm{x}} \mathrm{As}$," Journal of Applied Physics, vol. 58, no. 3, pp. R1-R29, 1985.

[19] Y. A. Burenkov, S. Y. Davydov, and S. P. Nikanorov, "Elastic properties of indium-arsenide," Soviet Physics.Solid State, vol. 17, p. 1446, 1975.

[20] D. N. Nichols, D. S. Rimai, and R. J. Sladek, "Elastic anharmonicity of inp: its relationship to the high pressure transition," Solid State Communications, vol. 36, no. 8, pp. 667-669, 1980.

[21] S. Adachi, Properties of Aluminium Gallium Arsenide, The Institution of Electrical Engineers, London, UK, 3rd edition, 1993.

[22] A. A. Balandin, E. P. Pokatilov, and D. L. Nika, "Phonon engineering in hetero- and nanostructures," Journal of Nanoelectronics and Optoelectronics, vol. 2, no. 2, pp. 140-170, 2007.

[23] A. Polian, M. Grimsditch, and I. Grzegory, "Elastic constants of gallium nitride," Journal of Applied Physics, vol. 79, no. 6, pp. 3343-3344, 1996.

[24] L. E. McNeil, M. Grimsditch, and R. H. French, "Vibrational spectroscopy of Aluminum Nitride," Journal of the American Ceramic Society, vol. 76, no. 5, pp. 1132-1136, 1993. 

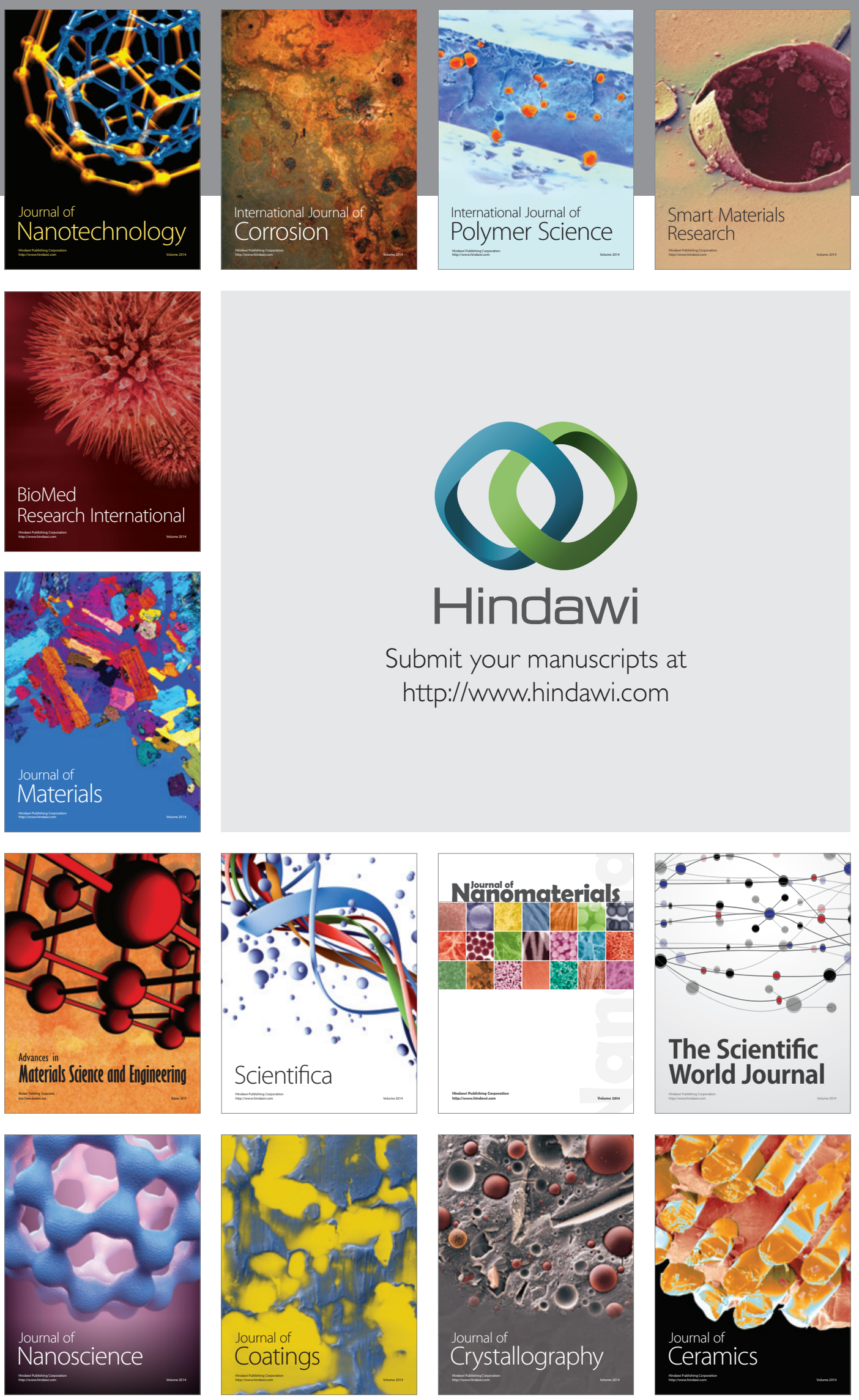

The Scientific World Journal

Submit your manuscripts at

http://www.hindawi.com

\section{World Journal}

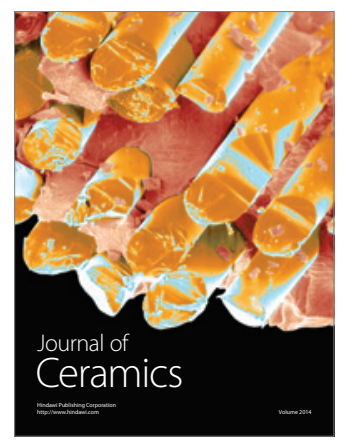

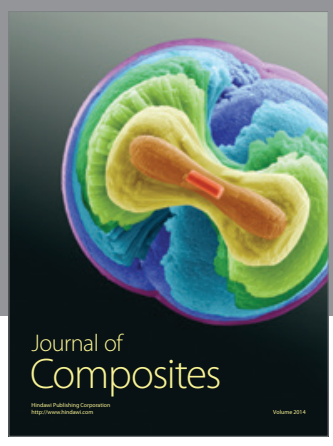
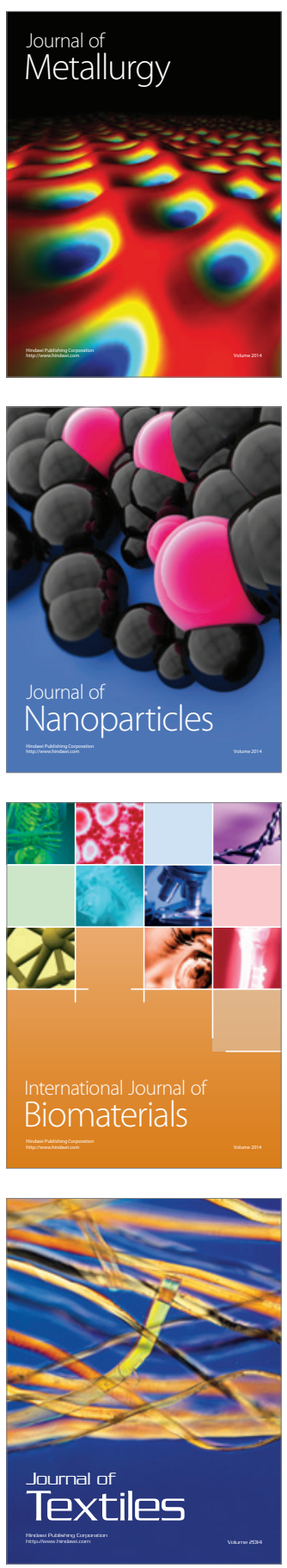